\section{Is there a need for new agents with novel mechanisms of action in psoriatic arthritis?}

\author{
Carl Orr, Douglas James Veale
}

The armoury of biological agents available to treat rheumatoid arthritis (RA) has expanded greatly over the last 15 years, but treatment options for psoriatic arthritis (PsA) have failed to keep pace. Therapy with disease modifying antirheumatic drugs (DMARDs), such as methotrexate and leflunomide, remain first-line agents despite a paucity of randomised controlled trial evidence. ${ }^{1}$ There is a good evidence base for the efficacy of TNF $\alpha$ inhibition (TNFi), both as monotherapy and in combination with methotrexate, from a number of studies. ${ }^{2-5}$ The use of TNFi has the additional benefit of efficacy in treating spinal symptoms where conventional DMARDs show little efficacy. ${ }^{6}$ Five TNFi agents are now approved for use in PsA patients in Europe, and all show equivalent efficacy in the treatment of joint disease. ${ }^{17-9}$

PsA is the second most common inflammatory arthropathy and represents a considerable disease burden for patients and the healthcare system. Therefore, the relative paucity of alternative agents in the treatment of PsA compared with RA represents a significant challenge. Moreover, PsA is not a benign disease, as evidenced by radiological progression and disability. ${ }^{10}$ In one study of PsA patients attending an early arthritis clinic, treatment with conventional DMARDs for 2 years achieved good disease control, however as many as $50 \%$ of PsA patients developed significant bone erosions in that time. ${ }^{11}$

Our understanding of the pathogenesis of PsA has significantly advanced in recent years, and this has led to the identification of various targets of the inflammatory process observed in PsA. ${ }^{12}$ It has been clear for some time that in psoriasis $(\mathrm{PsO})$ and PsA, there is a complex interaction of environmental and genetic factors that

Dublin Academic Medical Centre, Centre for Arthritis and Rheumatic Diseases, St Vincent's University Hospital and The Conway Institute of Biomolecular and Biomedical Research, University College Dublin, Dublin, Ireland

Correspondence to Professor Douglas J Veale, Dublin Academic Medical Centre, Centre for Arthritis and Rheumatic Diseases, St Vincent's University Hospital, Elm Park, Dublin 4, Ireland; douglas.veale@ucd.ie must lead to a sustained and persistent inappropriate inflammatory response. The relationship between skin and joint disease has been the focus of a great deal of research, and while it is clear that there are many common pathogenic features, the genetic associations may be independent. Environmental triggers have proven difficult to identify due to the heterogeneous nature of the disease(s). The debate continues, therefore, regarding whether $\mathrm{PsO}$ and PsA are separate diseases in their own right, or whether each clinical presentation represents part of a spectrum of the same disease. ${ }^{13}$

Undoubtedly, the most exciting and promising development in our understanding of the pathophysiology of PsA, since TNF, concerns the new targets to interrupt the IL-17 and IL-12/IL-23 pathway. There is evidence that IL-23 is essential for enthesitis to develop by acting on a specific $\mathrm{T}$ cell subset. Sherlock et $a l^{14}$ have demonstrated the presence of a very specific $\mathrm{T}$ cell subset (IL-23R(+), RAR-related orphan receptor $\gamma \mathrm{t}$ (ROR- $\gamma \mathrm{t}$ ) $(+) \mathrm{CD} 3(+) \mathrm{CD} 4(-) \mathrm{CD} 8(-)$, stem cell antigen $1(\mathrm{Sca} 1)(+))$ at the entheseal insertion in an animal model of enthesitis. The importance of this finding may have direct relevance to, and support, the targeting of this pathway in PsA.

TNFi therapies have offered a major been demonstrated in routine clinical practice that a remission rate of up to $60 \%$ is achievable. ${ }^{15}$ However, up to $30 \%$ of patients may demonstrate an inadequate or poor response and others may be unsuitable for, or intolerant of, such treatment. The European Medicines Agency (EMA) and the Food and Drug Administration (FDA) recognise that there are few alternative options for PsA patients who have failed TNFi therapy. It is in this relative 'treatment vacuum' that two further key studies of a novel biologic agent-ustekinumab-are reported in this issue of the journal.

In contrast to the treatment options for RA, which include several biological therapies targeting novel molecular pathways, there are few alternatives licensed for the treatment of PsA. In RA, B cells, advance in the treatment of PsA and it has interleukin-6 (IL-6) and the $\mathrm{T}$ cell co-stimulatory molecule CD80/86 (abatacept) have been shown to be relevant targets against which a number of biologic drugs are approved. In addition, new small molecular agents that target different aspects of the inflammatory pathway (eg, janus kinase signalling pathway), have been approved in a number of other countries, although not currently in Europe. In PsA, none of these agents have been approved and initial pilot studies show little relevance for B cells or IL-6 as a target. However, a number of novel targets are now proposed in PsA.

The two reports presented in this issue relate to the IL-12/IL-23 pathway, a novel target in PsA. Ustekinumab, a fully human monoclonal antibody, binds to the common p40 subunit of IL-12 and IL-23. IL-12 is a key cytokine in the Th1 inflammatory response, and IL-23 is involved in the activation of Th17 cells and the subsequent production of IL-17. The efficacy and safety of ustekinumab in patients with PsA naive to TNFi was firmly established in the landmark phase III PSUMMIT1 trial. Having examined the PSUMMIT1 trial together with the data from PSUMMIT 2, both the EMA and the FDA have approved ustekinumab for the treatment of PsA. ${ }^{16} 17$

PSUMMIT2 unequivocally demonstrates the clinical efficacy (in achieving ACR20 response) of ustekinumab in PsA patients who have not responded to TNFi, and therefore suggests that this drug may be a suitable alternative therapy in such patients. Although not entirely clear, it appears that most patients who have been included in the TNF-experienced arm of this study discontinued TNFi because it was ineffective (between $64 \%$ and $72 \%$ ). The majority of these patients had previously been on at least two TNFi, and 25\% had been on three TNFi. It is likely that this cohort represents more recalcitrant disease, or perhaps a subset of PsA patients whose disease is phenotypically distinct and not primarily driven by TNF. Just over one third $(35.6 \%)$ of patients who were TNFi experienced went on to achieve an ACR20 response, which is somewhat less than that seen in the TNFi naive patients in PSUMMIT1 $(46 \%)$ or PSUMMIT2 (54.4\%).

The PSUMMIT2 study is smaller than PSUMMIT1, but the design is very similar and it is appropriately powered to answer the clinical question. The primary endpoint remains as for PSUMMIT1 (the proportion of patients achieving ACR20 response at week 24), and the TNFi naive cohort for PSUMMIT2 was comparable 
to that enrolled in PSUMMIT1. Almost half of the patients in each arm of both studies were taking methotrexate.

With respect to safety endpoints, no new issues were raised in PSUMMIT2 compared to PSUMMIT1. Safety data are reported for longer in PSUMMIT2 (60 weeks) compared to PSUMMIT1 (52 weeks). Further safety data regarding ustekinumab are, of course, available from studies of its use in PsO. The EMA concluded that the evidence does not suggest any excess cardiovascular risk associated with ustekinumab treatment and that a minor increased incidence of depression is recognised, ${ }^{18}$ however the duration of the trials limits a definitive conclusion in relation to these issues long term. The same is true with respect to the question of whether ustekinumab increases the risk of malignancy. These questions should be addressed by long-term follow-up and post-marketing surveillance by the biologic registries. The EMA has licensed ustekinumab for use in PsA patients who have not responded to conventional DMARDs.

The question of radiographic progression in those treated with ustekinumab is also addressed in this issue. Combined radiographic results (at 24 weeks) are presented for both PSUMMIT1 and PSUMMIT2, demonstrating that ustekinumab decreases radiographic progression as measured by the PsA modified vdH-S scoring method, ranging from 0 to 528 points. ${ }^{19}$ The pre-planned integrated analysis is reported thus because the study required higher numbers to be enrolled to be appropriately powered to demonstrate this outcome.

The clinical relevance of minor radiographic progression (an increase in score over $6-12$ months of 1 or 2 points) is a matter of ongoing debate. Smolen et al ${ }^{20}$ have shown that in RA, an increase in the Sharp Score of a single point is approximately the equivalent of a change in the Health Assessment Questionnaire (HAQ) score of 0.01 . It is unlikely that such small changes in Sharp Scores would attain greater clinical relevance in PsA. Therefore, a change in the HAQ score of $0.19-0.23$ is required before a change in function is observed, ${ }^{21}$ and this means that an increase of 19-23 Sharp points is expected to be necessary before this would have a clinically meaningful effect. Whether other imaging modalities are more sensitive for erosive joint change is also hotly debated, and whether these other modalities in turn are in any way relevant to clinical impact on patients is a further unanswered question.
The study highlights challenges that are more general to rheumatology clinical research measuring outcomes of radiographic progression over relatively short time periods (eg, 24 weeks in this study). It also highlights some of the difficulties more peculiar to PsA specifically, and spondyloarthropathy in general, which has a wider spectrum of radiographic variability than other inflammatory arthropathies.

Nevertheless, progression of erosive disease is widely held to be most empirically measurable using radiographic scores of plain films. TNFi has been shown to be associated with a reduction in radiographic progression for PsA and it is therefore important that ustekinumab be shown to have a similar effect on radiographic progression.

Interestingly, in the sub-analysis of PSUMMIT2 data alone, the placebo and actively treated groups were not dissimilar with respect to radiographic progression. It is worth noting that more imputation of radiological data was required in the placebo arm, which may be a confounding factor. The numbers were also significantly smaller in PSUMMIT2 and the rules for data extrapolation probably conservatively favoured the placebo group. Van der Heijde et al have demonstrated recently how differing imputation methodology for missing data can affect the analysis and reporting of results of radiographic progression. This has been proven for the modified Total Sharp Score (mTSS) in a study of certolizumab. ${ }^{22}$

Monoclonal antibodies have been developed and their efficacy demonstrated in targeting the key IL-17 and IL-12/IL-23 regulatory pathway. Secukinumab is a fully human anti-IL-17A monoclonal antibody and in a small proof-of-concept study including 42 patients for 24 weeks, significant improvements in secondary measures were observed, although it failed to meet the primary endpoint. ${ }^{23}$ Two antibodies, brodalimumab targeting the IL-17 receptor, and ixekizumab targeting IL-17A, have also been shown in short phase II studies to be significantly beneficial in plaque PsO, however studies in PsA are awaited. ${ }^{24} 25$ There is already some evidence to support the use of abatacept to treat patients with PsA. ${ }^{26}$

More recently, several small molecular inhibitors are in development and will undoubtedly be exploited for the treatment of PsO and PsA. These include the oral phosphodiesterase 4 inhibitor apremilast and the janus kinase inhibitors. Of these, apremilast appears to be the most promising. The PALACE phase III trials compared two doses of apremilast with placebo in those with active disease despite prior treatment with DMARDs or biologics. The PALACE 1 trial has demonstrated that ACR20 responses of $40 \%$ at the higher dose (30 mg twice daily) can be achieved by week $16 .^{27}$ Long-term data for the PALACE 2 trial presented at the annual meeting of the American College of Rheumatology last year demonstrated ACR20 responses of $52 \%$ at 52 weeks. There were no new safety concerns, although tolerability due to gastrointestinal disturbances can be an issue in the short term. ${ }^{28}$

In conclusion, while TNFi biological agents remain the first choice biologic for the treatment of moderate to severe PsA, an unmet need has clearly been identified in those PsA patients who are not suitable, do not tolerate or do not respond to conventional DMARDs and/or TNFi. In this clinical circumstance, the results of the PSUMMIT studies together provide clear evidence for the efficacy of ustekinumab in the treatment of the signs and symptoms of PsA. The combined analysis also suggests that there is a benefit in inhibiting radiologic progression, although the data from the PSUMMIT2 sub-analysis are less conclusive. A head-to-head trial of TNFi versus ustekinumab would be useful to determine the best first choice biologic for patients with PsA.

\section{Competing interests None.}

Provenance and peer review Commissioned; externally peer reviewed.

To cite Orr C, Veale DJ. Ann Rheum Dis 2014;73:951-953.

Received 2 January 2014

Accepted 28 February 2014

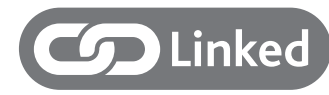

http://dx.doi.org/10.1136/annrheumdis-2013204655

- http://dx.doi.org/10.1136/annrheumdis-2013204741

Ann Rheum Dis 2014;73:951-953.

doi:10.1136/annrheumdis-2013-204934

\section{REFERENCES}

1 Mease P. Update on treatment of psoriatic arthritis Bull NYU Hosp Jt Dis 2012:70:167-71.

2 Kavanaugh A, Antoni CE, Gladman D, et al. The Infliximab Multinational Psoriatic Arthritis Controlled Trial (IMPACT): results of radiographic analyses after 1 year. Ann Rheum Dis 2006;65:1038-43.

3 Mease PJ, Goffe BS, Metz J, et al. Etanercept in the treatment of psoriatic arthritis and psoriasis: a randomised trial. Lancet 2000;356:385-90.

4 Antoni C, Krueger GG, de Vlam K, et al. Infliximab improves signs and symptoms of psoriatic arthritis: 
results of the IMPACT 2 trial. Ann Rheum Dis 2005;64:1150-7.

5 Mease PJ, Gladman DD, Ritchlin CT, et al. Adalimumab for the treatment of patients with moderately to severely active psoriatic arthritis: results of a double-blind, randomized, placebo-controlled trial. Arthritis Rheum 2005;52:3279-89.

6 Gossec L, Smolen J, Gaujoux-Viala C, et al. European League Against Rheumatism recommendations for the management of psoriatic arthritis with pharmacological therapies. Ann Rheum Dis 2012;71:4-12.

7 Ritchlin C, Kavanaugh A, Gladman D, et al. Treatment recommendations for psoriatic arthritis. Ann Rheum Dis 2009;68:1387-94.

8 Glintborg B, Østergaard M, Dreyer L, et al. Treatment response, drug survival, and predictors thereof in 764 patients with psoriatic arthritis treated with anti-tumor necrosis factor $\alpha$ therapy: results from the nationwide Danish DANBIO registry. Arthritis Rheum 2011;63:382-90.

9 Mease PJ, Fleischmann R, Deodhar AA, et al. Effect of certolizumab pegol on signs and symptoms in patients with psoriatic arthritis: 24-week results of a phase 3 double-blind randomised placebo-controlled study (RAPID-PsA). Ann Rheum Dis 2014;73:48-55.

10 Veale DJ. The epidemiology of psoriatic arthritis: fact or fiction? I Rheumatol 2000;27:1105-6.

11 Kane D, Stafford L, Bresnihan B, et al. A prospective, clinical and radiological study of early psoriatic arthritis: an early synovitis clinic experience. Rheumatology (Oxford) 2003;42:1460-8.

12 Veale DJ, Ritchlin C, FitzGerald O. Immunopathology of psoriasis and psoriatic arthritis. Ann Rheum Dis 2005;64(Suppl 2):ii26-9.

13 Ciocon DH, Kimball AB. Psoriasis and psoriatic arthritis: separate or one and the same? $\mathrm{Br} J$ Dermatol 2007;157:850-60.

14 Sherlock JP, Joyce-Shaikh B, Turner SP, et al. IL-23 induces spondyloarthropathy by acting on ROR- $\gamma \mathrm{t}+$
CD3+CD4-CD8-entheseal resident T cells. Nat Med 2012;18:1069-76.

15 Saber TP, Ng C, Renard G, et al. Remission in psoriatic arthritis: is it possible and how can it be predicted? Arthritis Res Ther 2010;12:R94.

16 Mclnnes IB, Kavanaugh A, Gottlieb AB, et al. Efficacy and safety of ustekinumab in patients with active psoriatic arthritis: 1 year results of the phase 3, multicentre, double-blind, placebo-controlled PSUMMIT 1 trial. Lancet 2013;382:780-9.

17 Ritchlin C, Rahman P, Kavanaugh A, et al. Efficacy and safety of the anti-IL-12/23 p40 monoclonal antibody, ustekinumab, in patients with active psoriatic arthritis despite conventional non-biological and biological anti-tumour necrosis factor therapy: 6-month and 1-year results of the phase 3 , multicentre, double-blind, placebo controlled, randomised PSUMMIT 2 trial. Ann Rheum Dis 2014;73:990-9.

18 Assessment Report: Stelara, Procedure No. EMEA/H/ C/000958/I//0029. European Medicines Agency, Committee for Medicinal Products for Human Use (CHMP). 25 July 2013.

19 Kavanaugh A, Ritchlin C, Rahman P, et al. Ustekinumab, an anti-IL-12/23 p40 monoclonal antibody, inhibits radiographic progression in patients with active psoriatic arthritis: results of an integrated analysis of radiographic data from the phase 3 , multicenter, randomized, double-blind, placebo controlled PSUMMIT-1 and PSUMMIT-2 trials. Ann Rheum Dis 2014;73:1000-6.

20 Smolen JS, Aletaha D, Grisar JC, et al. Estimation of a numerical value for joint damage-related physical disability in rheumatoid arthritis clinical trials. Ann Rheum Dis 2010;69:1058-64.

21 Redelmeier DA, Lorig K. Assessing the clinical importance of symptomatic improvements: an illustration in rheumatology. Arch Intern Med 1993;153:1337-42.
22 van der Heijde D, Fleischmann R, Wollenhaupt J, et al. Effect of different imputation approaches on the evaluation of radiographic progression in patients with psoriatic arthritis: results of the RAPID-PSA 24-week phase III double-blind randomised placebo-controlled study of certolizumab pegol. Ann Rheum Dis 2014;73:233-7.

23 McInnes IB, Sieper J, Braun J, et al. Efficacy and safety of secukinumab, a fully human anti-interleukin-17A monoclonal antibody, in patients with moderate-to-severe psoriatic arthritis: a 24-week, randomised, double-blind, placebo-controlled, phase II proof-of-concept trial. Ann Rheum Dis. 2014;73:349-56.

24 Papp KA, Leonardi C, Menter A, et al. Brodalumab, an anti-interleukin-17-receptor antibody for psoriasis. N Engl J Med 2012;366:1181-9.

25 Leonardi C, Matheson R, Zachariae C, et al. Anti-interleukin-17 monoclonal antibody ixekizumab in chronic plaque psoriasis. N Engl J Med 2012;366:1190-9.

26 Mease $\mathrm{P}$, Genovese MC, Gladstein G, et al. Abatacept in the treatment of patients with psoriatic arthritis: results of a six-month, multicenter, randomized, double-blind, placebo-controlled, phase II trial. Arthritis Rheum 2011;63:939-48.

27 Kavanaugh A, Mease P, Gómez-Reino JJ, et al. Treatment of psoriatic arthritis in a phase 3 randomized, placebo-controlled trial with apremilast, an oral phosphodiesterase 4 inhibitor. Ann Rheum Dis 2014;73:1020-6.

28 Cutolo M, Myerson GE, Fleischmann RM, et al. Long-term (52-week) results of a phase 3 , randomized, controlled trial of apremilast, an oral phosphodiesterase 4 inhibitor, in patients with psoriatic arthritis (PALACE 2). Arthritis Rheum 2013;65(Suppl):S346 